\title{
INTEGRABILITY CONDITIONS FOR VACUUM SPACETIMES WITH A KILLING VECTOR FIELD
}

\author{
FRANCESC FAYOS \\ Departament de Física Aplicada, UPC, E-08028 Barcelona, Spain \\ E-mail: labfm@ffn.ub.es \\ CARLOS F. SOPUERTA \\ Relativity and Cosmology Group, School of Computer Science and Mathematics, \\ Mercantile House, Hampshire Terrace, PO1 2EG Portsmouth, England \\ E-mail: carlos.sopuerta@port.ac.uk
}

\begin{abstract}
We present a new approach to the study of vacuum spacetimes with a Killing symmetry. The central quantity in this approach is the exterior derivative of the Killing vector field, which is a test electromagnetic field. Considering the algebraic structure of this quantity we get a new view of the integrability conditions, which provides a natural way of studying the connections between the algebraic structure of the spacetime and properties of the Killing symmetry.
\end{abstract}

Symmetries and in particular Killing symmetries have played an important role in the search and study of exact solutions of Einstein's equations. The main reason is that they lead to important simplifications of the equations. Another relevant way of simplifying Einstein's equations is to assume a special algebraic structure of the spacetime, which is determined by the Weyl tensor, and whose algebraic classification is the Petrov classification. However, very little is known about the connections between Killing symmetries and the algebraic structure of the spacetime.1 The aim of this communication is to present a new approach to the study of non-null KVFs in vacuum space-times which is suitable to study such connections.

The starting point is the fact, firstly noticed by Papapetrou, that, in vacuum, the exterior derivative of a Killing vector field (KVF) $\boldsymbol{\xi}$ is a 2 -form satisfying Maxwell's equations in the absence of electromagnetic currents

$$
\boldsymbol{F} \equiv \boldsymbol{d} \boldsymbol{\xi}, \quad \nabla_{[a} F_{b c]}=0, \quad \nabla_{b} F^{a b}=0 .
$$

The main object that we use in our study is the algebraic structure of $F_{a b}$. 3 There are only two different algebraic cases: (i) $F_{a b}$ is a regular 2 -form and hence it has two different principal null directions. (ii) $F_{a b}$ is a singular 2-form having only one (multiple) principal null direction. The case $F_{a b}=0$ corresponds either to the Minkowski spacetime or $p p$ waves (when the KVF is a null vector field).

The first application is to combine the algebraic structures of $F_{a b}$ and of the Weyl tensor $C_{a b c d}$ in order to classify space-times having at least one KVF, or more precisely, the pairs $\left\{\left(V_{4}, \boldsymbol{g}\right), \boldsymbol{\xi}\right\}$, which takes into account the fact that there are space-times with more than one KVF. Then, we can classify these pairs according to the following properties: The algebraic type of $F_{a b}$ [(i) or (ii) above]; the Petrov type of the space-time (I, II, III, D, N, or O); the degree of alignment of the principal directions of $F_{a b}$ with those of the Weyl tensor $C_{a b c d}$. Finally, we can refine this classification by adding differential invariant properties of the principal null directions of $F_{a b}$ and $C_{a b c d}$.

article: submitted to World Scientific on November 7, 2018 
The second application is to set up a new formalism for vacuum space-times with an isometry. The idea is to make an extension of the Newman-Penrose (NP) formalism, a formalism suitable to control the algebraic structure of the spacetime. This extension has two ingredients: (A) To write all the equations in a NP basis in which $F_{a b}$ takes its canonical form [(i) or (ii) above]. (B) To add the following variables and equations: The components of the KVF $\boldsymbol{\xi}$ and $F_{a b}$ in such a basis. The equations for these quantities are: the definition of $F_{a b}$ for the components of $\boldsymbol{\xi}$ and the Maxwell equations for the components of $F_{a b}$. Then, the alignment of a principal direction of $F_{a b}$ with one of $C_{a b c d}$ can be study in a natural way within this formalism. For instance, setting $\Psi_{0}=0$ we impose a principal direction of $F_{a b}$ to be aligned with one principal direction of the space-time.

In this formalism the integrability conditions for the components of the KVF determine completely the Weyl tensor as an algebraic combination of spin coefficients and quantities constructed from the KVF. As a consequence, we do not need to solve the second Bianchi identities, which are the equations for $C_{a b c d}$. Instead, we only have to substitute $C_{a b c d}$ in them, obtaining a set of consistency relations. Moreover, it turns out that the whole set of consistency relations and remaining integrability conditions constitute additional equations for the spin coefficients, which complement the Newman-Penrose equations. Therefore, we have to study their compatibility. The case in which $F_{a b}$ is singular has been completely examined $t$ and all the spacetimes and the KVFs determined: They correspond to two particular classes of $p p$ waves (Petrov type N solutions) and the Minkowski space-time. Currently we are studying the regular case. As an example, in the case of Petrov type III vacuum space-times we have arrived to the conclusion that the alignment of the multiple principal direction of $C_{a b c d}$ with any of the two principal directions of $F_{a b}$ is forbidden. In contrast to this situation, there are other vacuum space-times in which we can find alignments. An interesting example is the case of the Kerr metric in which the two multiple principal directions of the space-time (it is Petrov type D) are aligned with those of the Papapetrou field. 3

\section{Acknowledgments}

F.F. acknowledges financial support from the D.G.R. of the Generalitat de Catalunya (grant 1998GSR00015), and the Spanish Ministry of Education (contract PB96-0384). C.F.S. is supported by the European Commission (contract HPMF-CT-1999-00149).

\section{References}

1. D Kramer, H Stephani, M A H MacCallum and E Herlt, Exact solutions of Einstein's field equations (VEB Deutscher Verlag der Wissenschaften, Berlin, 1980).

2. A Papapetrou, Ann. Inst. H. Poincaré A4, 83 (1966).

3. F Fayos and C F Sopuerta, Class. Quant. Grav. 16, 2965 (1999).

4. F Fayos and C F Sopuerta, submitted to Class. Quant. Grav. (2000).

5. F Fayos and C F Sopuerta, in preparation (2000).

article: submitted to World Scientific on November 7, 2018 